\title{
A Cavity-Backed Slot Antenna on Timber
}

\author{
Mohamed Radwan \\ School of Engineering and Built \\ Environment \\ Griffith University \\ Brisbane, Australia \\ mohamed.radwan@griffithuni.edu.au
}

\author{
Yana Salchak \\ School of Engineering and Built \\ Environment \\ Griffith University \\ Brisbane, Australia \\ i.salchak@griffith.edu.au \\ Hugo G. Espinosa \\ School of Engineering and Built \\ Environment \\ Griffith University \\ Brisbane, Australia \\ h.espinosa@griffith.edu.au
}

\author{
Noor Albadri \\ School of Engineering and Built \\ Environment \\ Griffith University \\ Brisbane, Australia \\ n.al-badri@griffith.edu.au \\ David V. Thiel \\ School of Engineering and Built \\ Environment \\ Griffith University \\ Brisbane, Australia \\ d.thiel@griffith.edu.au
}

\begin{abstract}
Cavity-backed slot antennas can be used to detect anomalies and measure the dielectric properties of lossy media such as timber at microwave frequencies. An enhanced antenna design with the feed plate directly behind the rectangular slot allows maximum E-field coupling to the wood and provides more space for electronics inside the aluminum box. The E-field in a rectangular wood piece $(100 \mathrm{~mm} \times 70 \mathrm{~mm} \times 70 \mathrm{~mm})$ was calculated using CST simulations at the resonant frequency of 7.4 GHz. The wood material was modeled as isotropic with a relative permittivity of 2 and low conductivity $\left(10^{-6} \mathrm{~S} / \mathrm{m}\right)$. A comparison between simulations and a spherical wave calculation showed significant change in $E$-field in the very nearfield region of the antenna at the distances smaller than $4 \mathrm{~mm}$. The electrical field penetrated the $70 \mathrm{~mm}$ width, and reflection from the opposite wood-air interface was observed.
\end{abstract}

Keywords - slot antenna, non-destructive testing, structural timber, near field, microwave impedance.

\section{INTRODUCTION}

Microwave transmission has been used in testing wood for several years [1]. Timber is an anisotropic material and it is relatively transparent at microwave frequencies [2]. This opens an opportunity to study the anisotropic characteristics and dielectric properties of wooden beams at microwave frequencies using far-field measurements [1-4]. Saeidi et al. [5] applied near-field and far-field ultra-wideband (UWB) radio signals to image wood imperfections. Frequencies in the range of $1.25-1.35 \mathrm{GHz}$ and $1.7-1.81 \mathrm{GHz}$ were used in measurements of S-parameters (transmitted and received signals) performed in air, plywood and high-density wood. The results demonstrated that while the received signal amplitude changes, the pulse envelope does not change. This technique is adequate for imaging wooden beams when access is available to both sides of the wood with rectangular crosssection.

As reported in [6], three-dimensional numerical simulations using commercial software were performed to profile the electromagnetic field at $3 \mathrm{GHz}$ inside a cylindrical wood structure. The study reported the effect of the wood grain angle, the circular annual rings, and the effective permittivity of wood on the electric field distribution inside the wood cylinder.

A planar resonant slot antenna with a ground plane aperture was developed to determine the complex permittivity of a dielectric material in [7]. Part of the electric field permeates the dielectric substrate and enters into free space again through the slot. If an electric field penetrates a dielectric material (in this case wood), then computer simulations can reveal its behaviour inside that material for comparison with numerical calculations.

In this paper, a new feed for a slot antenna was designed and optimized for wood contact, based on a previously published antenna [8]. Experimental measurements and modelling using a cavity-backed slot antenna on concrete have also been used for electrical parameter measurements [9].The calculations and antenna design were conducted using the commercial software CST Microwave studio $($. The cavitybacked slot antenna was simulated as a rectangular box with a capacitively-coupled slot.

The slot dimensions and port position were adjusted to obtain the minimum $S_{11}$ values on pine wood with relative permittivity $\varepsilon_{\mathrm{r}}=2$. The $\mathrm{S}_{11}$ characteristics were adjusted by changing the relative permittivity in a previous model [10]. For a fixed relative permittivity of wood, a parameter sweep was applied in CST to determine the optimal location of the brass feed. At this position, a minimum reflection coefficient value was chosen to define the feed position and resonant frequency. The radiation pattern was determined inside the uniform pine wood beam based on simulations to calculate the electric field inside a wood piece with rectangular crosssection (dimensions $70 \times 70 \times 100 \mathrm{~mm}$ ).

\section{ANTENNA DESIGN}

To achieve a minimum $S_{11}$ at $50 \Omega$, the antenna impedance was matched to the surrounding media - in this case, wood. The feed was located on the side opposite the slot (see Fig. 1) and optimized to give the lowest $\mathrm{S}_{11}$ value.

The antenna slot placed on wood is resonant when the slot length is approximately equal to one half of the effective wavelength:

$$
l \cong \lambda / 2
$$

where $l$ is the slot antenna length and $\lambda$ is the effective wavelength. The effective wavelength depends on the free space wavelength and the effective wood permittivity, and it can be approximated by the equation [9]

$$
\lambda \cong \frac{\lambda_{0}}{\sqrt{\frac{1+\varepsilon_{r}}{2}}}
$$


where $\varepsilon_{r}$ is the relative permittivity of wood and $\lambda_{0}$ is the free space wavelength. For dry wood, the relative permittivity lies in the range from 1.5 to 4 [11]. In simulations, the wood was defined as a lossy media with a density of $454 \mathrm{~kg} / \mathrm{m}^{3}$, conductivity $\sigma$ of $10^{-6} \mathrm{~S} / \mathrm{m}$, and a relative permittivity of $2[11$, 12].

The electric field $E(X)$ close to the antenna aperture penetrates wood according to the equation $[13,14]$ :

$$
E(X)=E_{o} \frac{\exp (-\alpha X)}{X}
$$

where $E_{o}$ is the electric field strength on the surface of wood $(\mathrm{V} / \mathrm{m}), X$ is the distance $(\mathrm{m})$ from the surface of the wood and $\alpha$ is attenuation constant. By fitting the curve to equation (3), the attenuation constant $\alpha$ was calculated to be approximately $0.5 \mathrm{~Np} / \mathrm{m}$.

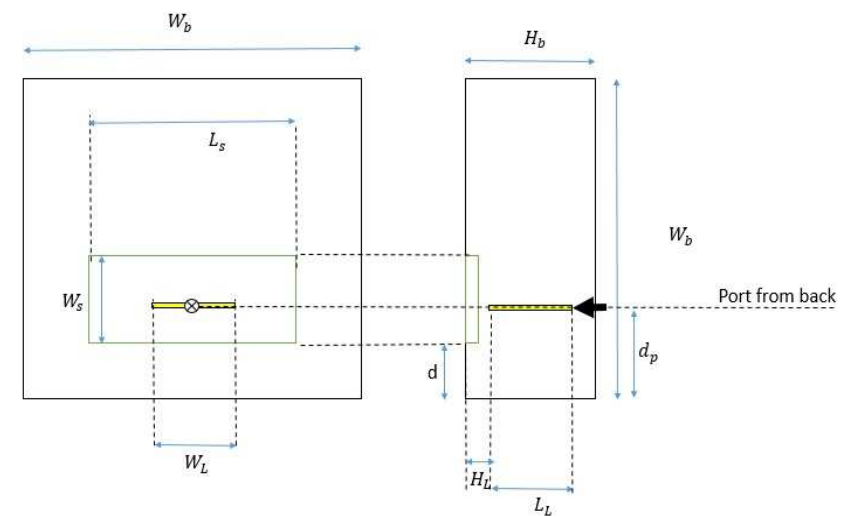

(a)

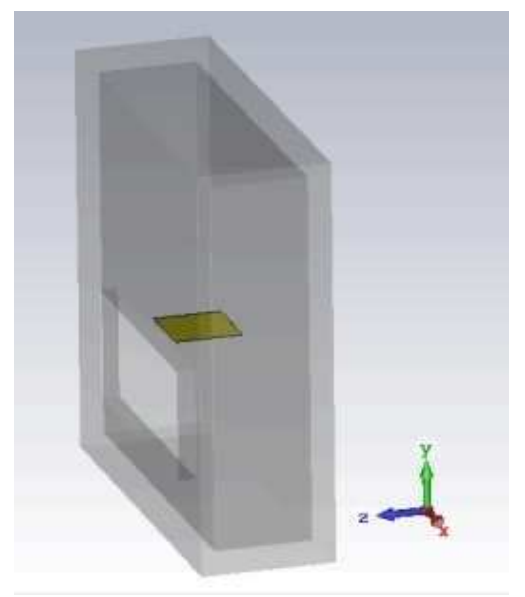

(b)

Fig. 1. (a) Top view (left) and side view of the cavity-backed slot antenna, (b) antenna model and brass position

\section{NUMERICAL SIMULATIONS}

The aluminum box $\left(W_{b} \times W_{b} \times H_{b}\right)$ has a slot $\left(W_{s} \times L_{s}\right)$ fed by a brass plate $\left(W_{L} \times L_{L}\right)$ located directly behind the slot. The antenna dimensions are given in Table I. The design challenge was to determine the brass position $\left(d_{p}\right)$ in Fig. 1 for a minimum $\mathrm{S}_{11}$ value with the slot against the flat surface of pine wood. By using CST parameter sweep function $\left(2<d_{p}<22\right.$ $\mathrm{mm}$ ), the minimum $\mathrm{S}_{11}$ value was found when $d_{p}=13 \mathrm{~mm}$ (see Fig. 2).
In CST, the antenna slot was placed against an isotropic pine woodblock $(70 \times 70 \times 100 \mathrm{~mm})$ with $454 \mathrm{~kg} / \mathrm{m}^{3}$ [11], and a relative permittivity $\varepsilon_{r}=2$. The resonant frequency of the antenna on wood was $7.4 \mathrm{GHz}$ with $\mathrm{S}_{11}=-45 \mathrm{~dB}$ (Fig. 3).

TABLE I. ANTENNA DIMENSION

\begin{tabular}{|c|l|l|l|l|l|l|l|l|l|}
\hline Parameter & $\mathbf{W}_{\mathbf{b}}$ & $\mathbf{W}_{\mathbf{s}}$ & $\mathbf{L}_{\mathbf{s}}$ & $\mathbf{L}_{\mathbf{L}}$ & $\mathbf{W}_{\mathbf{L}}$ & $\mathbf{d}_{\mathbf{P}}$ & $\mathbf{d}$ & $\mathbf{H}_{\mathbf{L}}$ & $\mathbf{H}_{\mathbf{b}}$ \\
\hline Value(mm) & 33 & 9 & 21 & 4.5 & 8 & 13 & 4 & 3.25 & 11 \\
\hline
\end{tabular}

Fig. 4 shows the comparison between CST results for $E$ field penetration inside the wood sample versus numerical calculations using (3) at $7.4 \mathrm{GHz}$ through the wood, directly in front of the center of the antenna aperture. These simulations show that the wood sample has a relatively large change in the very near-field region of the antenna (i.e., $X<4$ $\mathrm{mm})$. There is also evidence of reflection from the opposite wood-air interface creating a standing wave.

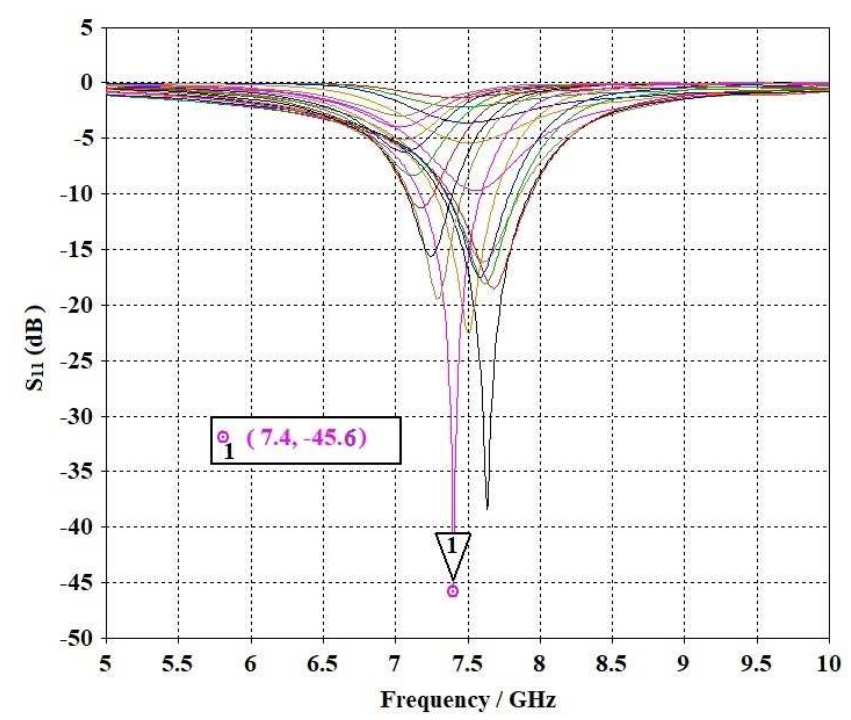

Fig. 2. $\mathrm{S}_{11}$ simulated values for various brass feed positions $d_{p}$ relative to the end of the cavity.

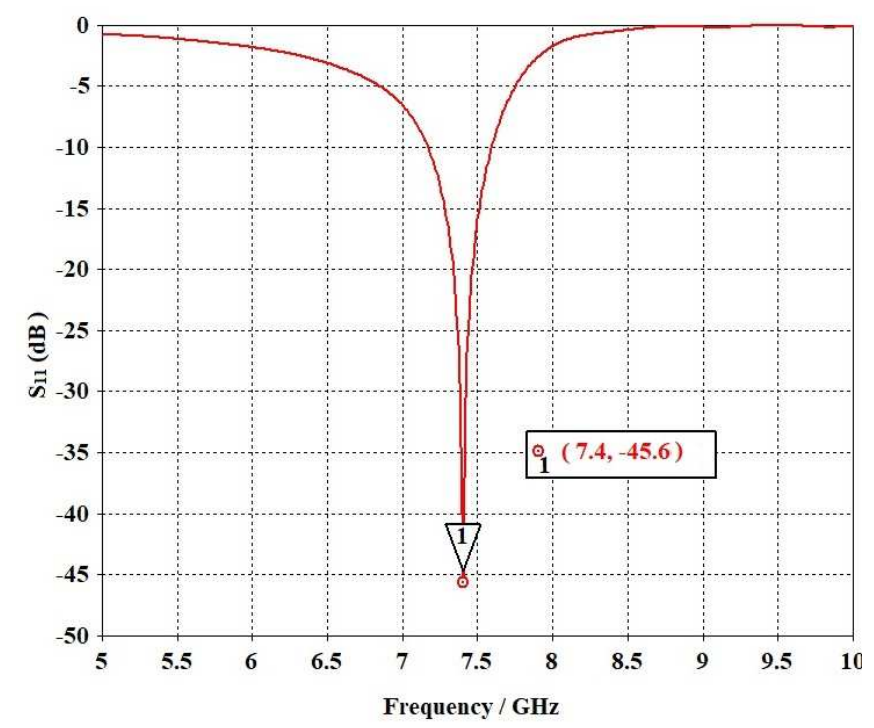

Fig. 3. Simulated $\mathrm{S}_{11}$ of the optimized antenna $\left(d_{p}=13 \mathrm{~mm}\right)$ against pine wood. The resonant frequency is $7.4 \mathrm{GHz}$. 
Related published work from other researchers [3] noted average values of relative permittivity at $9.8 \mathrm{GHz}$ for Poplar, Alder and Oriental beech woods between 1.1 and 4, depending on the moisture content. In the simulations presented in this paper, the relative permittivity was fixed at 2 and the attenuation constant $\alpha=0.5 \mathrm{~Np} / \mathrm{m}$ was relatively insignificant compared to the spherical wave field strength decrease.

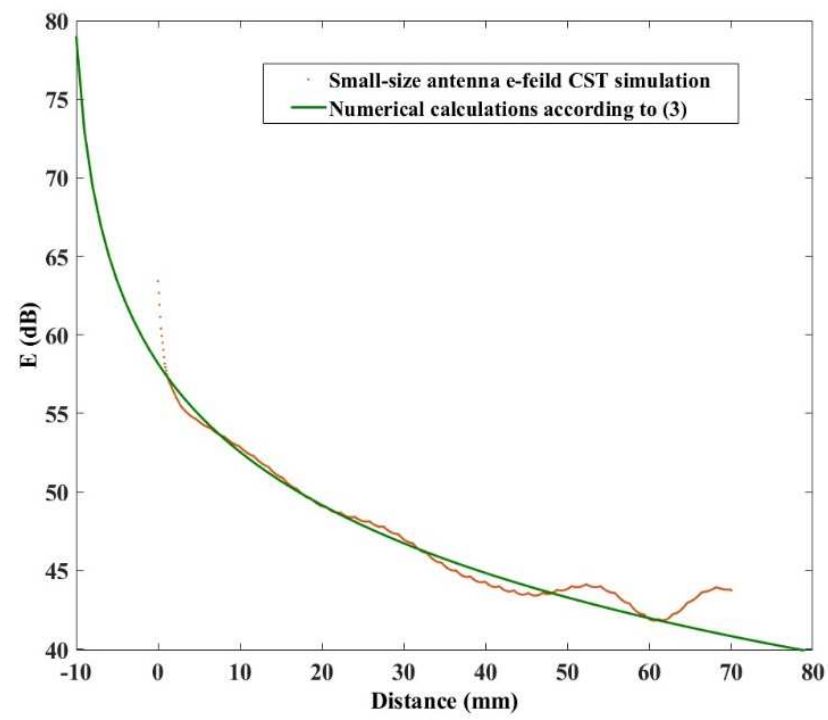

Fig.4. Simulated E-field on CST (orange line) vs numerical calculations according to (3) (green line). The surface of the wood is at distance zero.

\section{CONCLUSIONS}

The penetration depth of electromagnetic waves from a slot antenna on the surface of an isotropic woodblock, based on numerical calculations using CST, was approximately $4 \mathrm{~mm}$ from the wood surface. Beyond this distance, the field attenuates as an inverse square law as the conductivity is sufficiently low. Further work is required to explore the effect of wood anisotropy, the difference between wood types, the effect of moisture and finally the effect of discontinuities in wood beams.

\section{REFERENCES}

[1] M. Bogosanovic, A. Al Anbuky, and G.W. Emms, "Microwave nondestructive testing of wood anisotropy and scatter", IEEE Sensors, vol. 13 (1), pp. 306-313, doi: 10.1109/JSEN.2012.2211192, Aug. 2012.

[2] S. Ziherl, J. Bajc, B. Urankar, and M. Čepič, "Anisotropy of wood in the microwave region”, Eur. J. Phys., vol. 31, no. 3, pp. 531-542, doi: 10.1088/0143-0807/31/3/010, May 2010.

[3] H. Sahin and N. Ay, "Dielectric properties of hardwood species at microwave frequencies", J Wood Sci, vol. 50, no. 4, pp. 375-380, doi: 10.1007/s10086-003-0575-1, Aug. 2004.

[4] S. Ramasamy and B. Moghtaderi, "Dielectric properties of typical Australian wood-based biomass materials at microwave frequency", Energy \& Fuels, vol. 24, no. 8, pp. 4534-4548, doi: 10.1021/ef100623e, Aug. 2010.

[5] T. Saeidi, I. Ismail, A. R. H. Alhawari, and W. P. Wen, "Near-field and far-field investigation of miniaturized UWB antenna for imaging of wood", AIP Advances, vol. 9, no. 3, p. 035232, doi: 10.1063/1.5081762, Mar. 2019.

[6] R. Sanchez-Montero, P.-L. Lopez-Espi, J. Martinez-Rojas, J. AlpuenteHermosilla, and C. Alen-Cordero, "Numerical study of focusing effects of microwaves inside wood due to timber ring structure", Forests, vol. 9, no. 3, p. 106, doi: 10.3390/f9030106, Feb. 2018.

[7] D. Wylie, S.P. Rea, and D. Linton, "Non-destructive testing of complex permittivity using a slot antenna.", IEEE High Frequency Postgraduate Student Colloquium, Manchester, UK, pp.:31-36, doi: 10.1109/HFPSC.2004.1360344, 2004.

[8] N. Albadri, J. Hides, H.G. Espinosa and D.V. Thiel, "The effect of human tissue on field strength measurements in vivo using a resonant UHF cavity-backed slot antenna", Bioelectromagnetics, vol. 42 (4), pp. 284-295, 2021.

[9] A. A. Emelyanenko, J.-H. Doh, H. G. Espinosa, and D. V. Thiel, "Microwave measurements in cured concrete - Experiment and modelling", Australian Microwave Symposium (AMS), Brisbane, QLD, pp. 67-68. doi: 10.1109/AUSMS.2018.8346985, Feb. 2018.

[10] S. Higashi, and T. Fukusako, "A sensor antenna for non-destructive testing.", IEEE Conference on Antenna Measurements \& Applications (CAMA), Chiang Mai, Thailand, 2015.

[11] Timbeck, 'Resources | Timbeck | Premium Timber Finishes', Timbeck. https://timbeck.com.au/resources/ (accessed Jun. 07, 2021).

[12] F.T. Ulaby and U. Ravaioli, "Fundamentals of Applied Electromagnetics", $7^{\text {th }}$ ed. Pearson, Essex, UK, 2015.

[13] G.O. Torgovnikov, "Dielectric properties of wood-based materials", Springer, Berlin, Heidelberg, 1993.

[14] Y.A. Salchak, H.G. Espinosa, and D.V. Thiel, "Modelling the surface field from an ingested radio transmitter with an approximate attenuation model for gastroenterology investigations", IEEE Trans. $B M E$, vol. 67 (2), 2020. 